\title{
ON THE SYMPLECTIC EIGHTFOLD ASSOCIATED TO A PFAFFIAN CUBIC FOURFOLD
}

\author{
N. ADDINGTON AND M. LEHN
}

\begin{abstract}
We show that the irreducible holomorphic symplectic eightfold $Z$ associated to a cubic fourfold $Y$ not containing a plane is deformationequivalent to the Hilbert scheme of four points on a K3 surface. We do this by constructing for a generic Pfaffian cubic $Y$ a birational map $Z \rightarrow \operatorname{Hilb}^{4}(X)$, where $X$ is the K3 surface associated to $Y$ by Beauville and Donagi. We interpret $Z$ as a moduli space of complexes on $X$ and observe that at some point of $Z$, hence on a Zariski open subset, the complex is just the ideal sheaf of four points. This note is an appendix to [10].
\end{abstract}

\section{INTRODUCTION}

Beauville and Donagi [1] showed that if $Y \subset \mathbb{P}^{5}$ is a smooth cubic hypersurface then the variety $F$ of lines on $Y$ is an irreducible holomorphic symplectic fourfold. They did this by showing that for certain special cubics, called Pfaffian cubics, there is an associated $\mathrm{K} 3$ surface $X$ such that $F \cong \operatorname{Hilb}^{2}(X)$. Kuznetsov later observed that for a general $Y$, the $\mathrm{K} 3$ surface $X$ can be replaced with a "K3 category" $\mathcal{A}$, and he and Markushevich showed that $F$ is a moduli space of objects in $\mathcal{A}$, which in some sense explains the symplectic form on $F$. In more detail, the derived category $D(Y)=D^{b}(\operatorname{Coh}(Y))$ admits a semi-orthogonal decomposition

$$
D(Y)=\left\langle\mathcal{A}, \mathcal{O}_{Y}(-1), \mathcal{O}_{Y}, \mathcal{O}_{Y}(1)\right\rangle
$$

where $\mathcal{A}$ is like the derived category of a K3 surface in that it has the same Serre functor and Hochschild homology and cohomology, and $\mathcal{A} \cong D(X)$ if $Y$ is Pfaffian [7]. Given a line $\ell \subset Y$, the projection of the ideal sheaf $I_{\ell}$ into $\mathcal{A}$ is a stable sheaf whose deformation space is naturally identified with that of $\ell[9, \S 5]$.

Lehn et al. [10] associated to each cubic $Y$ not containing a plane an irreducible holomorphic symplectic eightfold $Z$, constructed not from lines but from twisted cubics on $Y$. They calculated that $Z$ has the same topological Euler number as $\mathrm{Hilb}^{4}(\mathrm{~K} 3)$, but left open the question of whether the two are deformation equivalent. In this note, using a derived interpretation like that of Kuznetsov and Markushevich, we show that they are.

Theorem - If $Y$ is a Pfaffian cubic fourfold not containing a plane and the associated K3 surface $X$ does not contain a line then $Z$ is birational to $\operatorname{Hilb}^{4}(X)$. 
Corollary — For any cubic fourfold $Y$ not containing a plane, $Z$ is deformation equivalent to the Hilbert scheme of four points on a K3 surface.

The corollary follows from Huybrechts' theorem that birational holomorphic symplectic varieties are deformation equivalent [4, Thm. 4.6].

In Section 1 we interpret $Z$ as a moduli space of objects in $\mathcal{A}$, clarifying the construction of [10]. There, $Z$ was constructed as a contraction of the moduli space $M$ of generalized twisted cubics on $Y$; precisely, there is an embedding $j: Y \rightarrow Z$ such that $M$ is a $\mathbb{P}^{2}$-bundle over the blow-up of $Z$ along $j(Y)$. Here we show that two points $\left[C_{1}\right],\left[C_{2}\right] \in M$ lie in the same fiber of $M \rightarrow Z$ if and only if the projections of the twisted ideal sheaves $I_{C_{1}}(2)$ and $I_{C_{2}}(2)$ into the subcategory $\mathcal{A}$ are the same, and that if $[C]$ lies over $j(y)$ then the projection of $I_{C}(2)$ is the same as the projection of the skyscraper sheaf $\mathcal{O}_{y}$, up to a shift.

In Section 2 we recall Beauville and Donagi's construction of a K3 surface $X$ associated to a Pfaffian cubic $Y$, and give a self-contained proof of Kuznetsov's equivalence $\mathcal{A} \cong D(X)$, emphasizing its geometric content: it is induced by the ideal sheaf of a correspondence $\Gamma \subset X \times Y$ that is generically 4-to-1 over $Y$.

In Section 3 we argue that if $Y$ is Pfaffian then for a general $[C] \in M$, the projection of $I_{C}(2)$ into $\mathcal{A} \cong D(X)$ is the ideal sheaf of four points in $X$, again up to a shift. Rather than proving this directly, we observe that if $[C]$ lies over $j(y)$ then we can instead pass $\mathcal{O}_{y}$ over to $D(X)$, and for generic $y$ this clearly yields the ideal sheaf of four points; but the property of being an ideal sheaf is an open condition. Thus we get a map from a Zariski open subset $M_{0} \subset M$ to $\operatorname{Hilb}^{4}(X)$, and from our work in Section 1 we see that this descends to an embedding of an open subset $Z_{1} \subset Z$ into $\operatorname{Hilb}^{4}(X)$.

After the preprint of this paper appeared, Ouchi [11] showed that for a very general $Y$ containing a plane - that is, in the opposite situation to [10] - there is a Bridgeland stability condition on $\mathcal{A}$ under which the projection of the skyscraper sheaf $\mathcal{O}_{y}$ into $\mathcal{A}$ is stable; hence $Y$ embeds as a Lagrangian in a holomorphic symplectic eightfold deformation-equivalent to $\mathrm{Hilb}^{4}(\mathrm{~K} 3)$. Presumably his eightfold is the limit of $Z$ as $Y$ approaches the locus of cubics containing a plane.

Acknowledgements. We thank E. Macrì and P. Stellari for discussing their related work in progress, A. Kuznetsov for discussing his, and J. Starr for informing us of his related unpublished work. We started this project at a workshop hosted by Y. Namikawa at the Research Institute for Mathematical Sciences in Kyoto, Japan, and finished it while the first author was visiting the Hausdorff Research Institute for Mathematics in Bonn, Germany; we thank both for their hospitality. Our travel was supported by SFB Transregio 45 Bonn-Mainz-Essen and NSF grant no. DMS-0905923. 


\section{1. $Z$ AS A MOdUli SPACE OF OBJECTS IN $\mathcal{A}$}

Let $M=\operatorname{Hilb}^{g t c}(Y)$ be the irreducible component of $\operatorname{Hilb}^{3 n+1}(Y)$ containing twisted cubics, and let $u: M \rightarrow Z$ be the contraction and $j: Y \rightarrow Z$ the embedding that appear in [10]. Recall from [10] that $u$ factors as $\sigma \circ a$, where $a: M \rightarrow Z^{\prime}$ is a $\mathbb{P}^{2}$-bundle and $\sigma: Z^{\prime} \rightarrow Z$ is the blow-up of $Z$ along $j(Y)$. The analysis of the curves $C$ parametrized by $M$ breaks into two cases, depending on whether $C$ is arithmetically Cohen-Macaulay (aCM) or non-Cohen-Macaulay (non-CM).

If $u([C]) \notin j(Y)$ then $C$ is aCM. The linear hull of $C$ is a $\mathbb{P}^{3}$, and the ideal sheaf of $C$ in this $\mathbb{P}^{3}$ has a resolution of the form

$$
0 \rightarrow \mathcal{O}_{\mathbb{P}^{3}}(-3)^{2} \rightarrow \mathcal{O}_{\mathbb{P}^{3}}(-2)^{3} \rightarrow I_{C / \mathbb{P}^{3}} \rightarrow 0 .
$$

Let $S_{C}=Y \cap \mathbb{P}^{3}$, which is a cubic surface. Any curve $C^{\prime}$ corresponding to a point in the same fiber $a^{-1}(a([C]))$ is contained in the same cubic surface $S_{C}$. Moreover there is a $3 \times 3$ matrix $A$ with entries in $H^{0}\left(S_{C}, \mathcal{O}(1)\right)$ such that for all such $C^{\prime}$ the ideal sheaf $I_{C^{\prime} / S_{C}}$ is generated by the minors of a $3 \times 2$ matrix $A_{0}$ consisting of two independent linear combinations of columns of $A$. Finally, $I_{C^{\prime} / S_{C}}$ admits a 2-periodic resolution

$$
\ldots \stackrel{A}{\longrightarrow} \mathcal{O}_{S_{C}}(-5)^{3} \stackrel{B}{\longrightarrow} \mathcal{O}_{S_{C}}(-3)^{\oplus 3} \stackrel{A}{\longrightarrow} \mathcal{O}_{S_{C}}(-2)^{3} \longrightarrow I_{C^{\prime} / S_{C}} \longrightarrow 0
$$

where $B$ is the adjugate matrix of $A$. In particular, as abstract sheaves, all $I_{C^{\prime} / S_{C}}$ for points $\left[C^{\prime}\right]$ in the same $a$-fiber are isomorphic. The converse holds as well: for any $\left[C^{\prime}\right] \in M$ with $I_{C^{\prime} / S_{C^{\prime}}} \cong I_{C / S_{C}}$ we have $\left[C^{\prime}\right] \in a^{-1}(a([C]))$. To see this, note that the curve $C$ can be reconstructed from its ideal sheaf by a choice of homomorphism $I_{C / S_{C}} \rightarrow \mathcal{O}_{S_{C}}$. From the resolution (1) and the exact sequence

$$
0 \rightarrow \mathcal{O}_{\mathbb{P}^{3}}(-3) \rightarrow I_{C / \mathbb{P}^{3}} \rightarrow I_{C / S_{C}} \rightarrow 0
$$

we find that $\operatorname{Hom}\left(I_{C / S_{C}}, \mathcal{O}_{S_{C}}\right)=H^{2}\left(I_{C / S_{C}}(-1)\right)^{*}$ is 3-dimensional, which gives a $\mathbb{P}^{2}$-family of distinct curves with isomorphic $I_{C / S_{C}}$. But the fiber $a^{-1}(a([C]))$ is already a $\mathbb{P}^{2}$-family of such curves, so there are no others.

If on the other hand $u([C])=j(y)$ then $C$ is non-CM, and consists of a singular plane cubic curve $C_{0} \subset S_{C}$ together with an embedded point at $y$. In particular $C$ has only one embedded point, so if two curves $C_{1}$ and $C_{2}$ both have embedded points at $y$ then $u\left(\left[C_{1}\right]\right)=u\left(\left[C_{2}\right]\right)=j(y)$.

This concludes our recollections from [10].

Let $L_{k}: D(Y) \rightarrow\left\langle\mathcal{O}_{Y}(k)\right\rangle^{\perp} \subset D(Y)$ be the left mutation past $\mathcal{O}_{Y}(k)$ :

$$
L_{k}(B)=\operatorname{cone}\left(\mathcal{O}_{Y}(k) \otimes \operatorname{RHom}\left(\mathcal{O}_{Y}(k), B\right) \rightarrow B\right) .
$$

Then the composition pr $:=L_{-1} \circ L_{0} \circ L_{1}$ is the projection into $\mathcal{A}$ discussed in the introduction. It annihilates $\mathcal{O}_{Y}(-1), \mathcal{O}_{Y}$, and $\mathcal{O}_{Y}(1)$, and acts as the identity on $\mathcal{A}$. It is left adjoint to the inclusion $\mathcal{A} \hookrightarrow D(Y)$. 


\section{Lemma 1}

(a) For all $[C] \in M$ one has $\operatorname{pr}\left(I_{C / Y}(2)\right) \cong \operatorname{pr}\left(I_{C / S_{C}}(2)\right)$.

(b) If $u([C])=j(y)$ then $\operatorname{pr}\left(I_{C / Y}(2)\right) \cong \operatorname{pr}\left(\mathcal{O}_{y}\right)[-1]$.

Proof. (a) From the Koszul resolution

$$
0 \rightarrow \mathcal{O}_{Y} \rightarrow \mathcal{O}_{Y}(1)^{2} \rightarrow I_{S_{C} / Y}(2) \rightarrow 0
$$

we see that that $\operatorname{pr}\left(I_{S_{C} / Y}(2)\right)=0$, so from the exact sequence

$$
0 \rightarrow I_{S_{C} / Y}(2) \rightarrow I_{C / Y}(2) \rightarrow I_{C / S_{C}}(2) \rightarrow 0
$$

we see that $\operatorname{pr}\left(I_{C / Y}(2)\right) \cong \operatorname{pr}\left(I_{C / S_{C}}(2)\right)$.

(b) Let $C_{0}$ be the singular plane cubic recalled above. From the Koszul resolution

$$
0 \rightarrow \mathcal{O}_{Y}(-1) \rightarrow \mathcal{O}_{Y}{ }^{3} \rightarrow \mathcal{O}_{Y}(1)^{3} \rightarrow I_{C_{0} / Y}(2) \rightarrow 0
$$

we see that $\operatorname{pr}\left(I_{C_{0} / Y}(2)\right)=0$, so from the exact sequence

$$
0 \rightarrow I_{C / Y}(2) \rightarrow I_{C_{0} / Y}(2) \rightarrow \mathcal{O}_{y} \rightarrow 0
$$

we see that $\operatorname{pr}\left(I_{C / Y}(2)\right) \cong \operatorname{pr}\left(\mathcal{O}_{y}\right)[-1]$.

Proposition $2-T$ wo points $\left[C_{1}\right],\left[C_{2}\right] \in M$ lie in the same fiber of $u: M \rightarrow Z$ if and only if $\operatorname{pr}\left(I_{C_{1}}(2)\right) \cong \operatorname{pr}\left(I_{C_{2}}(2)\right)$.

Proof. If $u\left(\left[C_{1}\right]\right)=u\left(\left[C_{2}\right]\right) \notin j(Y)$ then $a\left(\left[C_{1}\right]\right)=a\left(\left[C_{2}\right]\right)$, so $I_{C_{1} / S_{C_{1}}} \cong I_{C_{2} / S_{C_{2}}}$, so $\operatorname{pr}\left(I_{C_{1}}(2)\right) \cong \operatorname{pr}\left(I_{C_{2}}(2)\right)$ by Lemma $1\left(\right.$ a). If $u\left(\left[C_{1}\right]\right)=u\left(\left[C_{2}\right]\right)=j(y)$ then $\operatorname{pr}\left(I_{C_{1}}(2)\right) \cong \operatorname{pr}\left(I_{C_{2}}(2)\right)$ by Lemma $1(\mathrm{~b})$.

Conversely, suppose that $\operatorname{pr}\left(I_{C_{1}}(2)\right) \cong \operatorname{pr}\left(I_{C_{2}}(2)\right)$. We consider three cases.

Case 1: $C_{1}$ and $C_{2}$ are both aCM. It is enough to show that $\operatorname{pr}\left(I_{C / S_{C}}(2)\right)$ determines $I_{C / S_{C}}(2)$ for every aCM curve $C$. From (1) and (2) we find that $H^{*}\left(I_{C / S_{C}}\right)=H^{*}\left(I_{C / S_{C}}(1)\right)=0$, so $I_{C / S_{C}}(2) \in\left\langle\mathcal{O}_{Y}(1), \mathcal{O}_{Y}(2)\right\rangle^{\perp}$. Moreover we find that $I_{C / S_{C}}(2)$ is generated by global sections, so $F_{C}:=L_{0}\left(I_{C / S_{C}}(2)\right)[-1]$ is a sheaf and fits into an exact sequence

$$
0 \rightarrow F_{C} \rightarrow \mathcal{O}_{Y}^{3} \rightarrow I_{C / S_{C}}(2) \rightarrow 0 .
$$

As $I_{C / S_{C}}(2)$ has codimension 2 , dualizing this sequence gives $F_{C} \vee \cong\left(\mathcal{O}_{Y}^{3}\right)^{\vee}$, and dualizing again shows that the inclusion of $F_{C}$ in $\mathcal{O}_{X}^{3}$ is isomorphic to the natural map from $F_{C}$ to its double dual. Hence $I_{C / S_{C}}(2)$ can be recovered from $F_{C}$ as its cotorsion: $I_{C / S_{C}}(2) \cong F_{C} \vee \vee / F_{C}$. Now $F_{C}$ is contained in $\left\langle\mathcal{O}_{Y}, \mathcal{O}_{Y}(1), \mathcal{O}_{Y}(2)\right\rangle^{\perp}$. Since the canonical bundle $\omega_{Y}$ is $\mathcal{O}_{Y}(-3)$, the left mutation $L_{-1}$ and the corresponding right mutation $R_{-1}$ provide inverse equivalences

$$
\left\langle\mathcal{O}_{Y}, \mathcal{O}_{Y}(1), \mathcal{O}_{Y}(2)\right\rangle^{\perp} \underset{R_{-1}}{\stackrel{L_{-1}}{\rightleftarrows}}\left\langle\mathcal{O}_{Y}(-1), \mathcal{O}_{Y}, \mathcal{O}_{Y}(1)\right\rangle^{\perp}
$$

Hence $\operatorname{pr}\left(I_{C / S}(2)\right)=L_{-1}\left(F_{C}\right)$ determines $F_{C}$ and hence $I_{C / S}(2)$. 
ON THE SYMPLECTIC EIGHTFOLD ASSOCIATED TO A PFAFFIAN CUBIC FOURFOLD 5

Case 2: $C_{1}$ is aCM and $C_{2}$ is non-CM with embedded point $y_{2}$. Since pr is left adjoint to the inclusion $\mathcal{A} \hookrightarrow D(Y)$, we have

$$
\begin{aligned}
\operatorname{Hom}\left(\operatorname{pr}\left(I_{C_{1}}(2)\right), \operatorname{pr}\left(I_{C_{2}}(2)\right)\right) & =\operatorname{Hom}\left(\operatorname{pr}\left(I_{C_{1} / S_{C_{1}}}(2)\right), \operatorname{pr}\left(\mathcal{O}_{y_{2}}\right)[-1]\right) \\
& =\operatorname{Hom}\left(I_{C_{1} / S_{C_{1}}}(2), \operatorname{pr}\left(\mathcal{O}_{y_{2}}\right)[-1]\right)
\end{aligned}
$$

Applying pr to $\mathcal{O}_{y_{2}}[-1]$ we get a truncated Koszul complex

$$
\mathcal{O}_{Y}(-1)^{10} \rightarrow \mathcal{O}_{Y}{ }^{5} \rightarrow \underline{\mathcal{O}_{Y}(1)} \rightarrow \mathcal{O}_{y_{2}}
$$

where the underlined term is in degree zero. Applying $\operatorname{Hom}\left(I_{C_{1} / S_{C_{1}}}(2),-\right)$ to the complex (3) we find that the $E_{1}$ page of the Grothendieck spectral sequence is

$$
\begin{array}{ccccccc|c}
0 & \rightarrow & * & \rightarrow & * & \rightarrow & * & \\
0 & \rightarrow & * & \rightarrow & * & \rightarrow & * & \\
0 & \rightarrow & * & \rightarrow & * & \rightarrow & * & q=2 \\
0 & \rightarrow & 0 & \rightarrow & 0 & \rightarrow & * & q=1 \\
0 & \rightarrow & 0 & \rightarrow & 0 & \rightarrow & * & q=0 \\
\hline p=-2 & & p=-1 & & p=0 & & p=1 &
\end{array}
$$

where in the left-hand column we have used the fact that

$$
\operatorname{Ext}^{q}\left(I_{C_{1} / S_{C_{1}}}(2), \mathcal{O}_{Y}(-1)\right)=H^{4-q}\left(I_{C_{1} / S_{C_{1}}}\right)^{\vee}=0
$$

and the other zeroes come for dimension reasons. From this it follows that $\operatorname{Hom}\left(I_{C_{1} / S_{C_{1}}}(2), \operatorname{pr}\left(\mathcal{O}_{y_{2}}\right)[-1]\right)=0$, so $\operatorname{pr}\left(I_{C_{1}}(2)\right) \approx \operatorname{pr}\left(I_{C_{2}}(2)\right)$.

Case 3: $C_{1}$ and $C_{2}$ are non-CM with embedded points $y_{1}$ and $y_{2}$. We have

$$
\begin{aligned}
\operatorname{Hom}\left(\operatorname{pr}\left(I_{C_{1}}(2)\right), \operatorname{pr}\left(I_{C_{1}}(2)\right)\right) & =\operatorname{Hom}\left(\operatorname{pr}\left(\mathcal{O}_{y_{1}}\right), \operatorname{pr}\left(\mathcal{O}_{y_{2}}\right)\right) \\
& =\operatorname{Hom}\left(\mathcal{O}_{y_{1}}, \operatorname{pr}\left(\mathcal{O}_{y_{2}}\right)\right)
\end{aligned}
$$

By a similar Grothendieck spectral sequence calculation, this is $\operatorname{Hom}\left(\mathcal{O}_{y_{1}}, \mathcal{O}_{y_{2}}\right)$. Thus if $\operatorname{pr}\left(I_{C_{1}}(2)\right) \cong \operatorname{pr}\left(I_{C_{1}}(2)\right)$ then this Hom does not vanish, so $y_{1}=y_{2}$, so $u\left(\left[C_{1}\right]\right)=u\left(\left[C_{2}\right]\right)$.

Not only are the points of $Z$ in bijection with the objects $\operatorname{pr}\left(I_{C}(2)\right)$, but in fact the tangent spaces of $Z$ can be identified with the deformation spaces of the corresponding objects, so $Z$ truly deserves to be called a moduli space of objects in $\mathcal{A}$. But we will not prove this, as we do not need it for our main theorem.

Of course one would like to be able to define $Z$ directly as the component of the moduli space of stable objects in $\mathcal{A}$ containing $\operatorname{pr}\left(\mathcal{O}_{y}\right)[-1]$, thus avoiding the hard work of [10]. At present, though, no one knows how to produce any kind of stability condition on $\mathcal{A}$ when $Y$ is general. So while the derived perspective clarifies the construction of [10], it cannot yet replace it. 


\section{Pfaffian cubics}

Let $V$ be a 6-dimensional complex vector space and $L \subset \Lambda^{2} V^{*}$ a generic 6dimensional subspace of skew-symmetric forms on $V$. To these data Beauville and Donagi associate a K3 surface

$$
X=\left\{[P] \in \operatorname{Grass}(2, V)|\varphi|_{P}=0 \text { for all } \varphi \in L\right\}
$$

and a Pfaffian cubic fourfold

$$
Y=\left\{[\varphi] \in \mathbb{P}\left(L^{*}\right) \mid \operatorname{rk}(\varphi)=4\right\}=\left\{[\varphi] \in \mathbb{P}\left(L^{*}\right) \mid \operatorname{pf}(\varphi)=0\right\} .
$$

(Here we use Grothendieck's convention that $\mathbb{P}\left(L^{*}\right)$ is the space of 1-dimensional quotients of $L^{*}$, hence of 1-dimensional subspaces of $L$.) For a generic choice of $L$, both $X$ and $Y$ are smooth, and $X$ does not contain a line nor $Y$ a plane. Under this genericity assumption, $Y$ cannot contain a quadric surface either, for the linear hull of any quadric surface $Q \subset Y$ would cut out a residual plane.

In this section we study the correspondence

$$
\Gamma=\{([P],[\varphi]) \in X \times Y \mid P \cap \operatorname{rad}(\varphi) \neq 0\}
$$

and show that its ideal sheaf induces an equivalence between $D(X)$ and $\mathcal{A}$. This has been proved by Kuznetsov in [7] - see in particular the proof of his Lemma 8.2, where he shows that his kernel is $I_{\Gamma}$ twisted by a line bundle - but as his machinery is very heavy we prefer to give a self-contained account. The fiber of $\Gamma$ over a point of $X$ is a degree- 4 ruled surface on $Y$, as we shall see below; this family of surfaces is mentioned by Beauville and Donagi [1, Rem. (1)] and goes back to Fano [3].

The correspondence $\Gamma$ carries a natural scheme structure defined as follows: Let $0 \rightarrow \mathcal{P} \rightarrow V_{X} \rightarrow \mathcal{Q} \rightarrow 0$ denote the tautological bundle sequence on $X$, and let $A: V_{\mathbb{P}\left(L^{*}\right)} \rightarrow V_{\mathbb{P}\left(L^{*}\right)} \vee \otimes \mathcal{O}(1)$ denote the tautological skew-symmetric form parametrized by $\mathbb{P}\left(L^{*}\right)$. By construction, the restriction of $A_{\varphi}$ to any $P,[P] \in X$, vanishes, so $A$ induces a homomorphism $A^{\prime}: \mathcal{P} \otimes \mathcal{O} \rightarrow \mathcal{Q}^{\vee} \otimes \mathcal{O}(1)$ on $X \times \mathbb{P}\left(L^{*}\right)$. Then $\Gamma \subset X \times \mathbb{P}\left(L^{*}\right)$ is the subscheme defined by the vanishing of the $2 \times 2$-minors of $A^{\prime}$. There are natural morphisms $X \stackrel{p_{X}}{\longleftarrow} \Gamma \stackrel{p_{Y}}{\longrightarrow} Y$.

For any $[\varphi] \in Y$, the radical $\operatorname{rad}(\varphi)$ is a plane in $V$ which, however, can never lie in $X$ : In fact, up to a scalar factor, the differential $D_{\varphi}$ pf maps a tangent vector $\psi$ to its value on $\Lambda^{2} \operatorname{rad}(\varphi)$. As $Y$ is smooth, the intersection of $\mathbb{P}\left(L^{*}\right)$ and the Pfaffian hypersurface at $[\varphi]$ is transversal. Hence not all $\varphi^{\prime} \in L$ can vanish on $\operatorname{rad}(\varphi)$.

Thus the fiber $\Gamma_{P}:=p_{X}^{-1}([P])$ admits a well-defined map $\pi: \Gamma_{P} \rightarrow \mathbb{P}(P), \varphi \mapsto$ $P \cap \operatorname{rad}(\varphi)$. The fiber $\pi^{-1}([\ell])$ over a line $\ell \subset P$ is a linear subspace in $Y$. But by assumption, $Y$ does not contain a plane. Hence this fiber is at most 1-dimensional, and in turn $\operatorname{dim}\left(\Gamma_{P}\right) \leq 2$ and $\operatorname{dim}(\Gamma) \leq 4$. As $\Gamma$ is a determinantal variety, there is the a priori bound $\operatorname{codim}\left(\Gamma / X \times \mathbb{P}\left(L^{*}\right)\right) \leq 3$. We conclude that $\Gamma$ has the expected dimension 4, and that the Eagon-Northcott complex associated to $A^{\prime t}$ is a locally free resolution of the ideal sheaf $I_{\Gamma / X \times \mathbb{P}\left(L^{*}\right)}$, and $\Gamma$ is Cohen-Macaulay 
ON THE SYMPLECTIC EIGHTFOLD ASSOCIATED TO A PFAFFIAN CUBIC FOURFOLD 7

(cf. [2, Thm. A2.10 and Cor. A2.13]). Restricting the complex to $[P] \times \mathbb{P}\left(L^{*}\right)$, we obtain a locally free resolution

$$
0 \rightarrow \mathcal{O}_{\mathbb{P}\left(L^{*}\right)}(-4)^{3} \rightarrow \mathcal{O}_{\mathbb{P}\left(L^{*}\right)}(-3)^{8} \rightarrow \mathcal{O}_{\mathbb{P}\left(L^{*}\right)}(-2)^{6} \rightarrow \mathcal{O}_{\mathbb{P}\left(L^{*}\right)} \rightarrow \mathcal{O}_{\Gamma_{P}} \rightarrow 0
$$

In particular, the Hilbert polynomial of $\Gamma_{P}$ is constant as a function of $P$, and $p_{X}: \Gamma \rightarrow X$ is flat. Moreover, each $\Gamma_{P}$ is a 2-dimensional Cohen-Macaulay subscheme of $Y$ of degree 4 . Since $Y$ does not contain planes or quadric surfaces, $\Gamma_{P}$ is generically reduced and hence reduced.

Let $\Phi: D(Y) \rightarrow D(X)$ be the Fourier-Mukai functor induced by the ideal sheaf $I_{\Gamma}=I_{\Gamma / X \times Y}$, and let $\Psi: D(X) \rightarrow D(Y)$ be its right adjoint. From the resolution (4) and the exact sequence

$$
0 \rightarrow \mathcal{O}_{\mathbb{P}\left(L^{*}\right)}(-3) \rightarrow I_{\Gamma_{P} / \mathbb{P}\left(L^{*}\right)} \rightarrow I_{\Gamma_{P} / Y} \rightarrow 0
$$

we find that $\Phi\left(\mathcal{O}_{Y}(k)\right)=0$ for $k=-1,0,1$. This implies that $\Psi(D(X)) \subset \mathcal{A}$.

Proposition 3 (Kuznetsov) - $\Psi: D(X) \rightarrow \mathcal{A}$ is an equivalence.

Proof. Step 1. For distinct points $[P],[Q] \in X$, the corresponding subvarieties $\Gamma_{P}$, $\Gamma_{Q} \subset Y$ are distinct: We have $P \cap Q=0$; otherwise $X$ would contain a line. Hence if $\Gamma_{P}=\Gamma_{Q}$, the mappings $[\varphi] \mapsto \operatorname{rad}(\varphi) \cap P$ and $[\varphi] \mapsto \operatorname{rad}(\varphi) \cap Q$ would define two different rulings on $\Gamma_{P}=\Gamma_{Q}$, which is impossible.

Step 2. The functor $\Psi$ is fully faithful: By the criterion of Bondal and Orlov [5, Prop. 7.1], it is enough to show that

$$
\operatorname{dim} \operatorname{Ext}_{Y}^{i}\left(\Psi\left(\mathcal{O}_{[P]}\right), \Psi\left(\mathcal{O}_{[Q]}\right)\right)=\operatorname{dim} \operatorname{Ext}_{X}^{i}\left(\mathcal{O}_{[P]}, \mathcal{O}_{[Q]}\right) .
$$

The kernel inducing $\Psi$ is $I^{\vee} \otimes \mathcal{O}_{Y}(-3)[4]$ (cf. [5, Prop. 5.9]). Since $\Gamma$ is flat over $X$ we have $\Psi\left(\mathcal{O}_{[P]}\right)=I_{\Gamma_{P} / Y}^{\vee}(-3)[4]$, so we can rewrite (5) as

$$
\operatorname{dim} \operatorname{Ext}_{Y}^{i}\left(I_{\Gamma_{Q} / Y}, I_{\Gamma_{P} / Y}\right)=\operatorname{dim}_{\operatorname{Ext}_{X}}^{i}\left(\mathcal{O}_{[P]}, \mathcal{O}_{[Q]}\right)
$$

This is trivial for $i<0$ and obviously true for $i=0$, since $\Gamma_{P}$ and $\Gamma_{Q}$ have codimension 2 in $Y$ and are distinct if $P \neq Q$. Kuznetsov [8, Cor. 4.4] has shown that the Serre functor of $\mathcal{A}$ is given by shifting by 2. Thus Serre duality gives the claim for $i \geq 2$. Finally, Hirzebruch-Riemann-Roch gives $\chi\left(I_{\Gamma_{Q} / Y}, I_{\Gamma_{P} / Y}\right)=0$, so the claim also holds in the remaining case $i=1$.

Step 3. Since $\Psi$ is fully faithful and the Serre functor is given by shifting by 2 on both $D(X)$ and $\mathcal{A}$, it is enough to show that $\mathcal{A}$ is indecomposable [5, Cor. 1.56]. This follows from the fact that $H H^{0}(\mathcal{A}) \cong H H_{-2}(\mathcal{A})$ is 1-dimensional [6].

Lemma 4 - The projection $p_{Y}: \Gamma \rightarrow Y$ is generically finite of degree 4 .

Proof. Fix $[\varphi] \in Y$. Then the fiber $\Gamma_{\varphi}:=p_{Y}^{-1}([\varphi])$ is a linear section of the Schubert cycle

$$
\Sigma_{\varphi}:=\{[Q] \in \operatorname{Grass}(2, V) \mid Q \cap \operatorname{rad}(\varphi) \neq 0\}
$$


which is 5-dimensional. Choose a basis $\varphi_{1}, \ldots, \varphi_{6}$ of $L$ with $\varphi_{1}=\varphi$. This determines 6 hyperplane sections $\varphi_{1}^{\perp}, \ldots, \varphi_{6}^{\perp}$ of $\operatorname{Grass}(2, V)$, and $\Gamma_{\varphi}=\Sigma_{\varphi} \cap \varphi_{1}^{\perp} \cap \cdots \cap \varphi_{6}^{\perp}$. But $\Sigma_{\varphi}$ is already contained in $\varphi_{1}^{\perp}=\varphi^{\perp}$, so $\Gamma_{\varphi}$ is the intersection of $\Sigma_{\varphi}$ with 5 hyperplanes, hence is non-empty. Since $\operatorname{dim} \Gamma=\operatorname{dim} Y$, we see that $p_{Y}$ is generically finite. With a bit of Schubert calculus we find that $\operatorname{deg} \Sigma_{\varphi}=4$, so when $\Gamma_{\varphi}$ has the expected dimension it is a 0 -dimensional scheme of length 4.

In fact one can show that if $X$ contains no (-2)-curves then $p_{Y}: \Gamma \rightarrow Y$ is flat, but we do not need this.

\section{THE BIRATIONAL ISOMORPHISM}

Now we assemble the results from the previous two sections to prove the theorem stated in the introduction. As in the previous section, let $Y$ be a Pfaffian cubic and $X$ the associated $\mathrm{K} 3$ surface, and assume that $X$ does not contain a line nor $Y$ a plane. All our pullbacks, pushforwards, etc. are implicitly derived.

Let $\mathcal{C} \subset M \times Y$ be the universal curve, and let $T$ be the convolution

$$
T:=I_{\Gamma} \circ I_{\mathcal{C}}(2)=\pi_{M \times X *}\left(\pi_{X \times Y}^{*} I_{\Gamma} \otimes \pi_{M \times Y}^{*} I_{\mathcal{C}}(2)\right) \in D(M \times X) .
$$

For each $[C] \in M$, let $i_{[C]}: X \rightarrow M \times X$ be the inclusion $x \mapsto([C], x)$. Because $\mathcal{C}$ is flat over $M$, the derived restriction $i_{[C]}^{*} T$ is isomorphic to $\Phi\left(I_{C}(2)\right)$.

By Lemma 4 , there is an open subset $Y_{0} \subset Y$ such that $\Phi\left(\mathcal{O}_{y}\right)$ is the ideal sheaf $I_{\xi(y) / X}$ of a 0 -dimensional subscheme $\xi(y) \subset X$ of length 4 for all $y \in Y_{0}$. Since $\Phi$ annihilates $\mathcal{O}_{Y}(-1), \mathcal{O}_{Y}$, and $\mathcal{O}_{Y}(1)$, we have $\Phi \circ \mathrm{pr}=\Phi$, so by Lemma $1(\mathrm{~b})$, the sheaves $\mathcal{H}^{k}\left(i_{[C]}^{*} T\right)$ vanish for $k \neq 1$ whenever $u([C]) \in j\left(Y_{0}\right)$. By semicontinuity, the same then holds for all $[C]$ in an open neighborhood $M_{0}$ of $u^{-1}\left(j\left(Y_{0}\right)\right)$. Hence by [5, Lem. 3.31], the sheaf $E:=\mathcal{H}^{1}\left(\left.T\right|_{M_{0} \times X}\right)$ is flat over $M_{0}$, and $\left.T\right|_{M_{0} \times X} \cong E[-1]$. Over $u^{-1}\left(j\left(Y_{0}\right)\right) \subset M_{0}$ the family $E$ parametrizes ideal sheaves on $X$, and since ideal sheaves are stable, we conclude after shrinking $M_{0}$ if necessary that $E$ is an $M_{0}$-flat family of ideal sheaves on $X$.

Let $t^{\prime}: M_{0} \rightarrow \operatorname{Hilb}^{4}(X)$ be the classifying morphism induced by the family $E$. Proposition 2 implies that $t^{\prime}$ is constant on the fibers of $u$. As $u$ is proper, there is an open neighborhood $Z_{0}$ of $j\left(Y_{0}\right)$ in $Z$ such that $u^{-1}\left(Z_{0}\right) \subset M_{0}$. The restriction $T_{u^{-1}\left(Z_{0}\right)}^{\prime}$ now descends to give a morphism $t: Z_{0} \rightarrow \operatorname{Hilb}^{4}(X)$.

It follows from Proposition 3 that $\Psi \circ \Phi=$ pr, so by Proposition 2 again we see that $t$ is injective. The differential of $t$ must have full rank at some point otherwise $t\left(Z_{0}\right)$ would be a proper subscheme of $\operatorname{Hilb}^{4}(X)$, contradicting injectivity - and hence it must have full rank on an open subset of $Z_{1}$ of $Z_{0}$. Now $\left.t\right|_{Z_{1}}$ is injective and étale, hence is an open immersion. Thus $Z$ is birational to $\operatorname{Hilb}^{4}(X)$. 
ON THE SYMPLECTIC EIGHTFOLD ASSOCIATED TO A PFAFFIAN CUBIC FOURFOLD 9

\section{REFERENCES}

[1] A. Beauville and R. Donagi. La variété des droites d'une hypersurface cubique de dimension 4. C. R. Acad. Sci. Paris Sér. I Math., 301(14):703-706, 1985. Also math1.unice.fr/ beauvill/pubs/bd.pdf.

[2] D. Eisenbud. Commutative algebra, volume 150 of Graduate Texts in Mathematics. SpringerVerlag, New York, 1995. With a view toward algebraic geometry.

[3] G. Fano. Sulle forme cubiche dello spazio a cinque dimensioni contenenti rigate razionali del $4^{\circ}$ ordine. Comment. Math. Helv., 15:71-80, 1943.

[4] D. Huybrechts. Compact hyper-Kähler manifolds: basic results. Invent. Math., 135(1):63113, 1999. Also alg-geom/9705025.

[5] D. Huybrechts. Fourier-Mukai transforms in algebraic geometry. Oxford Mathematical Monographs. The Clarendon Press Oxford University Press, Oxford, 2006.

[6] A. Kuznetsov. Hochschild homology and semiorthogonal decompositions. Preprint, arXiv:0904.4330.

[7] A. Kuznetsov. Homological projective duality for Grassmannians of lines. Preprint, math/0610957.

[8] A. Kuznetsov. Derived category of a cubic threefold and the variety $V_{14}$. Tr. Mat. Inst. Steklova, 246(Algebr. Geom. Metody, Svyazi i Prilozh.):183-207, 2004. Also math/0303037.

[9] A. Kuznetsov and D. Markushevich. Symplectic structures on moduli spaces of sheaves via the Atiyah class. J. Geom. Phys., 59(7):843-860, 2009. Also math/0703264.

[10] C. Lehn, M. Lehn, C. Sorger, and D. van Straten. Twisted cubics on cubic fourfolds. J. reine angew. Math., to appear. Also arXiv:1305.0178.

[11] G. Ouchi. Lagrangian embeddings of cubic fourfolds containing a plane. Preprint, arXiv:1407.7265.

E-mail address: adding@math.duke.edu

Department of Mathematics, Duke University, Box 90320, Durham, NC 27708-0320, UNITED STATES

E-mail address: lehn@mathematik.uni-mainz.de

Institut für Mathematik, Johannes Gutenberg-Universität Mainz, 55099 Mainz, GerMANY 